\title{
SUICIDAL IDEATION IN ADOLESCENTS: A REVIEW OF RELATED STUDIES
}

\author{
Anumeha1, Dr. (Fr.) Ignatius Topno S.J ${ }^{2}$ \\ Research Scholar, School of Educational Training and Research, Aryabhatta Knowledge University, Patna, Bihar ${ }^{1}$ \\ Department of Education, St. Xavier's College of Education (Autonomous), Patna ${ }^{2}$
}

\begin{abstract}
Adolescence is a critical period for the development of healthy behaviors and lifestyles. Suicide in young people is a serious and prevalent problem. It is the 3rd leading cause of death for young people of ages 10-24 and accounts for $20 \%$ of all deaths annually. Suicidal ideation means thoughts of suicide that can range in severity from a vague wish to be dead to active suicidal plan and intent. This article is a review of relevant literature concerning the interplay of psychological factors including depression, self-esteem, sexual abuse, lack of coping and socio demographic variables like age, sex, family types, place of residence. occupational status, income, present living arrangement, family size contributing to suicidal behaviour. Findings suggest that the psychological factors and socio demographic factors have significant impact on the suicidal ideation of adolescents.
\end{abstract}

Keywords : Suicidal Ideation, Adolescence, Psychological, Socio Demographic, Self Esteem

\section{INTRODUCTION}

Suicidal ideation may be defined as the thoughts of suicide that can range in severity from a vague wish to be dead to active suicidal ideation with a specific plan and intent. It is defined as a personality disorder. Suicidal ideation is defined as the common medical term for thoughts about suicide, which may be as detailed as a suicide plan, without the suicidal act itself; although most people who undergo suicidal ideation do not commit suicide to completion, a considerable proportion do go on to make suicide attempts People in the grip of suicidal ideation often feel that life isn't worth living. It ranges in intensity from fleeting thoughts through to concrete, well thought-out plans for killing oneself, or a complete preoccupation with self-destruction or self-harm.

Adolescent suicide attempts may be associated with feelings of stress, self- doubt, pressure to succeed, financial uncertainty, disappointment and loss. There may be some symptoms of suicide in individuals to which the parents and other related persons should give due attention to manage the affected cases on time. It may include threatening to hurt $\mathrm{him} /$ herself or suicide, looking for ways to suicide e.g. seeking access to pills, weapons, or other means, deliberately hurting him/herself i.e. by scratching, cutting, or burning. talking or writing about death, dying or suicide, hopelessness, rage, anger, seeking revenge, acting recklessly or engaging in risky activities, seemingly without thinking, feeling trapped, like there's no way out, increasing alcohol or drug use, withdrawing from friends, family or society, anxiety, agitation, changes in sleep or appetite, dramatic changes in mood, no reason for living, and no sense of purpose in life etc ( Silva et al.,2014; Schweitzer er al., 1995).

\section{NEED AND SIGNIFICANCE OF THE STUDY}

There has been a drastic elevation in the rate of suicides over the past 50 years The statistics show that adolescents aged between 13 and 19 years old are at high risk of committing suicide. This could be attributed to issues relating to the developmental stage of adolescents. During this stage, adolescents face challenges and are exposed to various stressful experiences and risk factors relating to suicide. On this preliminary background, the aim of the current study is to provide a systematic review of original studies by focusing on the psychological and socio demographic factors leading to suicidal ideation.

\section{OBJECTIVES}

The objectives of the present study are divided into two categories as Psychological factors and Socio demographic factors a) To study the influence of psychological risk factors such as presumptive stressful life events, self esteem, social support , family interaction pattern.

b) To study the influence of socio-demographic variables, such as age, sex, family types, place of residence. occupational status, income, present living arrangement, family size contributing to suicidal behaviour. 


\section{International Advanced Research Journal in Science, Engineering and Technology \\ Impact Factor 7.105 Vol. 9, Issue 1, January 2022 \\ DOI: 10.17148/IARJSET.2022.9139}

\subsection{SUICIDAL IDEATION AND PSYCHOLOGICAL FACTORS}

Guo, Y. \& et.al. (2021). studied the relationship between suicidal ideation and parental attachment among adolescents. The aim of this study was to explore how parental attachment, anhedonia, and peer attachment were associated with suicidal ideation in adolescents. The study suggested that parental attachment could directly influence suicidal ideation and indirectly influence suicidal ideation via anhedonia and peer attachment. The results emphasized the importance of attachment in infancy and verified the feasibility of intervention on anhedonia and peer attachment to prevent suicidal ideation.

Campisi, S. C.\& et. al (2020) conducted a study on suicidal behaviours among adolescents from 90 countries The prevalence of suicidal ideation representing 397,299 adolescents (51.3\% female) was significantly higher among girls than boys whereas attempts did not differ by age or sex. Being bullied, or having no close friends was associated with suicidal ideation among girls 13-15 years and 16-17 years, respectively. Among all boys, being in a fight and having no close friends was associated with suicidal ideation with the addition of serious injury for boys 13-15 years. Common to all younger adolescents was an association of suicide attempt with being bullied and having had a serious injury. Among young boys, having no close friends was an additional indicator for suicide attempt. Having no close friends was associated with suicide attempt in older adolescents with the addition to being bullied in older girls and serious injury in older boys. Building positive social relationships with peers and avoiding serious injury appear key to suicide prevention strategies for vulnerable adolescents. Targeted programs by age group and sex for such indicators could improve mental health during adolescence in low and middle-income countries, given the diverse risk profiles for suicidal ideation and attempts.

Upadhyay and Singh (2017) investigated on Suicide Ideation and Psychopathology Among Adolescents. Aim of the present investigation was to know the differences between male and female adolescents. Study revealed that male adolescents scored significantly higher on the measures of suicidal ideation, whereas male adolescents had more psychological impairment on psychopathic deviation. There was significance gender difference on the measure of suicide ideation.

Silva et.al (2014) pointed out some trajectories to follow in the observation, mapping, and intervention on suicidal ideation. They pointed to the need for actions aimed at adolescents, especially females, and greater attention to violent behavior and illicit drug use as a major concern for the prevention of suicide among males.

Jason (2014) conducted a study on Suicidal Behaviour and Related Risk Factors among School-Aged Youth in the Republic of Benin. The survey indicated that $23.2 \%$ had thought about suicide and $28.3 \%$ had made a suicide attempt in the previous year. Anxiety, loneliness, being bullied, alcohol misuse, illicit drug use, and lack of parental support were independently related to the ideation outcomes, suicidal ideation without planning and suicidal ideation with planning. Multinomial regression analysis, using one suicide attempt and multiple suicide attempts as outcomes, revealed that female sex, anxiety, loneliness, being physically attacked, and illicit drug use were associated these outcomes.

Manani and Sharma (2013) studied the relationship between self-esteem and suicidal ideation and found that there was negative correlation between self- esteem and suicidal ideation among students. A negative view of the self may involve seeing the self as worthless and the future as hopeless. This type of feeling gives rise to suicidal ideation and also to the suicidal attempts.

Peltzer and Pengpid (2012) conducted a study on Suicidal Ideation and Associated Factors among School-Going Adolescents in Thailand. The aim of this study was to estimate the prevalence and identify associations between suicidal ideation and indicators of psychosocial distress and social-environmental factors in Thai adolescents. Psychosocial, health-risk behaviours and lack of protective factors appeared to effect suicidal ideation in this youth population.

Creemers, et al. (2012) examined whether explicit and implicit self-esteem, the interaction between these two constructs, and their discrepancy are associated with depressive symptoms, suicidal ideation, and loneliness. They found that the interaction of implicit and explicit self-esteem was associated with suicidal ideation, indicating that participants with high implicit selfesteem combined with a low explicit self-esteem showed more suicidal ideation.

Man Ng, Sheng Ran and Chan (2010) studied on Factors Related to Suicidal Ideation among Adolescents in Hong Kong. The study investigated the relations among suicidal ideation, general mental health status (domains: depression, lack of confidence and uselessness; measured by the shorter General Health Questionnaire [GHQ]), and psychosocial difficulties and strengths with 2638 secondary school students in Hong Kong. A cross-sectional survey using self-report questionnaires was carried out. Suicidal ideation The prevalence of suicidal ideation revealed in this study was lower than findings of other local studies which included non-school participants.

\subsection{SUICIDAL IDEATION AND SOCIO DEMOGRAPHIC FACTORS}

Quarshie, E.NB., Odame, S.K.(2021) aimed to estimate the 12-month prevalence of suicidal ideation and to describe some of the common and gender-specific associated factors among in-school adolescents in rural Ghana. Females who experienced personal and interpersonal adversities mainly outside the family context were likely to report suicidal 


\section{International Advanced Research Journal in Science, Engineering and Technology \\ Impact Factor $7.105 \div$ Vol. 9, Issue 1, January 2022 \\ DOI: 10.17148/IARJSET.2022.9139}

ideation, while suicidal ideation among males was associated with conflict with parents. Regardless of gender, adolescents who reported exposure to a friend's attempted suicide were about two times more likely to report suicidal ideation.

Ibrahim, N., Amit, N., Che Din, N., \& Ong, H. C. (2017). studied on gender differences and psychological factors associated with suicidal ideation among youth in Malaysia. The objective of this study was to compare gender differences in suicidal ideation and determine the predictors of suicidal ideation among youth. This cross-sectional study was carried out among 232 youths aged between 15 and 25 years from selected urban areas in Malaysia. The results showed that suicidal ideation was higher among male participants compared with female participants. Age was the predictor of suicidal ideation for males, while depression and loss of motivation, as components of hopelessness, were the predictors of suicidal ideation among females. Hence, it is important that professionals conduct early identification tests for suicidality among young people. This will facilitate the early detection of depression and hopelessness, which is important, in order to prevent suicidal behaviors or other problems before these occur.

Ziaei et.al.(2017) had conducted a study to determine the prevalence and correlates of suicidal ideation among 15-17year-old high school students in Iran. The results of the study showed that Overall, 62 of 1,517 students had thoughts of suicide. Three hundred and thirteen students reported being bullied in the previous 30 days. In addition, 134 students reported having been sexually abused. Being worried that they could not eat or did not feel hungry; thinking about using alcohol or other drugs and being sexually abused were all factors positively associated with suicidal ideation. The study concluded that the prevalence of suicidal ideation was lower in school students than in earlier studies.

Hollinger (2016) conducted a study on Adolescent Attitudes Toward and Perceptions of Suicide, Stigma, and HelpSeeking Behavior. Results indicated that an overwhelming majority of participants believe that suicide can be prevented and that intervention is necessary; however, adolescents still hold some degree of stigmatizing attitudes toward suicide and are uncertain about how to identify and assist those at risk. There were few significant differences between the perceptions and attitudes of males and females on this topic.

Singh and Sharma (2016) explored on Suicide Ideation and Life Satisfaction among Adolescents. The results of the study revealed that girls as compared to boys scored higher on Suicide Ideation whereas boys scored higher than girls on different components of life satisfaction such as family, friends and living environment. The study also explored a negative relationship between Suicide Ideation and different dimensions of life satisfaction such as family, friends, self, living environment as well as overall life satisfaction in both the genders.

Gupta (2015) conducted a study to determine the prevalence of suicide ideation and to study its predictive factors among school going adolescents. The findings of the study revealed that a total of 218 study subjects had suicide ideation. Discussing problems with parents, having good relations with school teachers and helpful classmates lowered the odds of having suicidal ideations. On the contrary, adolescents having worrying issues in family, verbally or physically abused and body image conscious had increased odds of suicidal ideations. The conclusions of the study showed that Suicidal ideation is a common experience among adolescents residing in Shimla district of North India. The supportive environment at home and in school decrease its vulnerability.

Shawashereh (2015) investigated on the suicidal ideation in relation to the total level of self-esteem among first year junior students. The results indicated a low level of suicidal ideation among university students. More specifically, factors that influenced students' suicidal ideation were identified as those of psychological, sociological, physical health, family in addition to economic ones. Furthermore, the study reported that there was a significant positive relationship between suicidal ideation and the self-esteem level among students. As such, students with high self-esteem obtained highest suicidal ideation scores.

Arya (2015) studied on Suicide: Knowledge and attitude among higher secondary school adolescents in Alwar, Rajasthan. Findings suggested that there was no significant association found between adolescents' socio-sample variables except source of information with knowledge and adolescent's socio-sample variables except sex, parental education, family pattern, socioeconomic status, habit/ substance use \& personal experience with attitude towards suicide.

Ahmad ( 2014) focused on Suicidal Ideation Among Malaysian Adolescents. This study aimed to identify the risk and protective factors associated with suicidal ideation among Malaysian adolescents. Analysis revealed that suicidal ideation was positively associated with depression, anxiety, stress, substance use, being bullied, and being abused at home, either physically or verbally. In addition, suicidal ideation was significantly higher among females and among the Indians and Chinese. Having close friends and married parents were strongly protective against suicidal ideation. Understanding the risk and protective factors is important in providing comprehensive management for suicidal ideation.

Kumar, Dixit and Kenswar (2014) conducted a study on Racial differences in suicidal ideation among school going adolescents. The aim of this study was to assess suicidal ideation in school going tribal and nontribal adolescents and to study its relationships with psychological well-being, depression, and anxiety. There were no racial differences in suicidal ideation and psychological discomfort among tribal and nontribal adolescents. Tribal adolescents, and more specifically tribal boys, had more depression than their nontribal counterparts. Suicidal ideation was positively correlated with psychological discomfort, anxiety, and depression 


\section{International Advanced Research Journal in Science, Engineering and Technology \\ Impact Factor $7.105 \div$ Vol. 9, Issue 1, January 2022 \\ DOI: 10.17148/IARJSET.2022.9139}

Smith (2014) investigated on the effects of physical activity on suicidal ideation. The study examines how physical activity influences suicidal ideation longitudinally in a population-based sample of adolescents in grades 7 through 12, as well as investigates potential mediating and moderating factors of this association. This study included measures of physical activity, self-esteem, social support, and suicidal ideation. The findings of this study illustrate that physical activity served as a protective factor of suicidal ideation in adolescents, though this effect may vary according to gender. Silva, Santos, Soares and Pardono ( 2014) studied on suicidal Ideation and Associated Factors among Adolescents in Northeastern Brazil. This study aimed to identify the prevalence and factors associated with suicidal ideation among Brazilian adolescents. The findings of the study showed an association between being female and suicidal ideation , suicide planning, and suicide attempt was found. Violent behavior/involvement in fights was associated with thinking about suicide, suicide planning, and suicide attempt .For cigarette consumption, association was found with suicide ideation, planning and attempt. For alcohol consumption, association was found with suicide ideation, planning, and attempt . It was concluded that suicidal ideation was associated with female sex, involvement in fights, and illicit drug use.

Shilubane et.al, (2014) focused on High school students' knowledge and experience with a peer who committed or attempted suicide: a focus group study. Results of the study revealed that Participants reported to be affected by the suicide attempt or completed suicide felt guilty about their failure to identify and prevent the suicide and displayed little knowledge of warning signs for suicidal behaviour. They identified several risk factors for the suicide of their peers, such as poor relationship issues, teenage pregnancy, punishment, and attention seeking behaviour. Resources for students with mental health problems and survivors of suicide attempts were not perceived to be available at schools and elsewhere.

Cederbaum et.al, (2013) studied on Well-Being and Suicidal Ideation of Secondary School Students From Military Families. The study sought to better understand the influence of parental military connectedness and parental deployment on adolescent mental health. Findings emphasize the increased risk of mental health issues among youth with parents (and siblings) in the military. Although deployment-related mental health stressors are less likely during peace, during times of war there is a need for increased screening in primary care and school settings. Systematic referral systems and collaboration with community-based mental health centers will bolster screening and services.

Tuzla and Bajraktervic (2010) conducted a study on Depression and suicide ideation among secondary school adolescents involved in school bullying. Findings of the study revealed that there was an increased prevalence of depression and suicidal ideation in adolescents who have been victims in relationships to respondents who were uninvolved subjects. There was an increased prevalence of depression and suicidal ideation in adolescents who have been bully victims in relationships to respondents who were uninvolved subjects too. Adolescents who are victims and those who are bully victims are more likely to have suicide ideation compared to uninvolved subjects. Discontent with financial situation is a vulnerability factor associated with elevated levels of depression in victims.

\section{FINDINGS}

a) Studies revealed that, anxiety, loneliness, being physically attacked, and illicit drug use were associated with suicidal ideation.

b) Anxiety, loneliness, being bullied, alcohol misuse, illicit drug use, and lack of parental support were independently related to the ideation outcomes.

c) Psychosocial, health-risk behaviours and lack of protective factors appeared to effect suicidal ideation

d) The level of suicidal ideation appears to increase with age, while socio-economic status, the influence of parents and peer romantic relationships all seem to contribute to the level of suicidal ideation

e) There is a negative relationship between Suicide Ideation and different dimensions of life satisfaction such as family, friends, self, living environment as well as overall life satisfaction in both the genders.

f) The supportive environment at home and in school decreases suicidal ideation.

\section{DISCUSSION}

1. Many commonly cited risk factors for suicide, including depression, hopelessness, most mental disorders, and impulsivity, are best conceptualized as predictors of suicidal ideation.

2. These same risk factors struggle to differentiate those who have attempted suicide from those who have experienced suicidal ideation without making an attempt.

3. The ideation-to-action framework stipulates that (a) the development of suicidal ideation and (b) the progression from ideation to attempts should be viewed as distinct processes with distinct predictors and explanations and should guide the next generation of suicide research, theory, and prevention.

4. The capacity to attempt suicide (including the capacity to tolerate the fear of pain and death that accompanies suicide attempts) plays a key role in the progression from ideation to attempts. 


\title{
International Advanced Research Journal in Science, Engineering and Technology \\ Impact Factor $7.105 \div$ Vol. 9, Issue 1, January 2022 \\ DOI: 10.17148/IARJSET.2022.9139 \\ CONCLUSION
}

\begin{abstract}
Although many researches have been conducted with respect to suicidal ideation in adolescents yet more number of studies are needed in this area so that suicidal ideation can be prevented in the adolescents. The correlates of suicide ideation examined in the study included bullying victimization, alcohol use, illicit drug use, having no friends, feeling sad, missing school, physical fighting and hunger. there is a need for increased research in the area of suicide and suicide related behaviors. Previous studies indicate that suicide ideation is strongly associated with certain risk factors. This study analyzed eight of these risk factors using a secondary logistical regression analysis of data from the Global Student Health Survey which is conducted annually by the WHO. The risk factors included in the study are bullying victimization, alcohol use, illicit drug use, having no friends, feeling sad, missing school, physical fighting and hunger. The results of this study may be useful to those looking to design and implement educational suicide prevention programs among schoolage children The research presented in this study may prove useful in determining which areas of suicide related risk factors are the most important to address when designing programs and interventions for adolescents.
\end{abstract}

\section{LIMITATIONS}

There are limitations when one conducts a literature review. The first limitation deals with investigator bias. When choosing articles, there is a tendency to select articles that support the theme of the literature review. Contrary information was not intentionally left out; however, contrary articles may not have been pursued as diligently. Thus, the result may have been that some of the data from related studies may not have been reported. The second limitation comes from the nature of the study. It is simply just a literature review. Although it is important to compile the relevant research on a common topic, it would be better to complete an experiment and further psychology's knowledge base with empirical data

\section{REFERENCES}

1.Ahmad, N., Cheong, S. M. Ibrahim, N. \&Rosman, R. (2014).Suicidal ideation among Malaysian adolescents. AsiaPacific Journal of Public Health, 26,(5), 63-69.

2.Campisi, S. C., Carducci, B., Akseer, N., Zasowski, C., Szatmari, P., \& Bhutta, Z. A. (2020). Suicidal behaviours among adolescents from 90 countries: a pooled analysis of the global school-based student health survey. BMC public health, 20(1), 1102. https://doi.org/10.1186/s12889-020-09209-z

3.Creemers, D. H. M., Scholte, R. H. J., Engels, R. C. M. E., Prinstein, M. J., \&Wiers, R. W. (2012). Implicit and explicit self-esteem as concurrent predictors of suicidal ideation, depressive symptoms, and loneliness. Journal of Behaviour Therapy and Experimental Psychiatry, 43 (1), 638-646.

4.Guo, Y., Ji, Y., Huang, Y., Jin, M., Lin, Y., Chen, Y., Zhang, L., Zhu, C., Yu, F., \& Wang, K. (2021). The Relationship Between Suicidal Ideation and Parental Attachment Among Adolescents: The Mediator of Anhedonia and Peer Attachment. Frontiers in psychology, 12, 727088. https://doi.org/10.3389/fpsyg.2021.727088

5.Hewitt, John P. (2009). Oxford Handbook of Positive Psychology. Oxford University Press. pp. 217-224.

6.Ibrahim, N., Amit, N., Che Din, N., \& Ong, H. C. (2017). Gender differences and psychological factors associated with suicidal ideation among youth in Malaysia. Psychology research and behavior management, 10, $129-135$. https://doi.org/10.2147/PRBM.S125176

7.Mallett, C., DeRigne, L. A., Quinn, L., \& Stoddard-Dare, P. (2012). Discerning reported suicide attempts within a youthful offender population. Suicide and Life-Threatening Behavior, 42(1), 67-77.

8.Manani, P. \& Sharma, S. (2013). Self- esteem and suicidal ideation : A Correlational study.MIER Journal of Educational Studies, Trends \& Practices, 3(1)75-78

9.Orth U.,\& Robbins R.W. (2014).The development of self-esteem. Current Directions in Psychological Science. 23 (5): 381-387.

10.Park, S. (2013). Gender-specific factors of suicide ideation among adolescents in the Republic of Korea: A nationally representative population-based study," Archives of Psychiatric Nursing, vol. 27, no. 5, pp. 253-259.

11.Pathak, N. \& R.N. Singh (2016). Adolescent suicidality and demographic risk factors. In Upadhyaya, S., Jaiswal, A.K. \& Singh, R. (2016).Psychology and Society Today: Contemporary researches. pp 149-159. MPS, Sanjai Nagar Colony, Chowkaghat, Varanasi

12.Silva, R.J., Santos, B.A., Soares, N. M. \&Pardono , E. (2014) Suicidal ideation and associated factors among adolescents in Northeastern Brazil. The Scientific World Journal ,2014 Article ID 450943, 8

13.Singh R N, \& Pathak N (2017). Effects of Self- esteem on Suicidal Ideation among Adolescents. International Journal of Indian Psychology, Vol. 4, (4), DIP:18.01.127/20170404, DOI:10.25215/0404.127

14.Souzal, L. D. M. ,Ores,L. II, \& de Oliveira G. T. II, et al., (2010). Ideaçãosuicidanaadolescência: prevalência e fatoresassociados. JornalBrasileiro de Psiquiatria,59,(4), 286-292, 2010. 
DOI: 10.17148/IARJSET.2022.9139

15. Yagual, S. (2015).Efectos de la violenciaintrafamiliaren el autoestima de losestudiantes de octavo y novenoaño de la Escuela de educaciónbásica 11 de Diciembre. Editorial La Libertad. Universidad EstatalPenínsula de Santa Elena, . Ecuador.

16.Thakur, D., Gupta, A., Thakur, A., Mazta, S. R., \& Sharma, D. (2015). Prevalence and predictors of suicidal ideations among school going adolescents in a hilly state of India. Industrial Psychiatry Journal, 24(2), 140-143. http://doi.org/10.4103/0972-6748.181719 https://www.ncbi.nlm.nih.gov/pmc/articles/PMC4866340/

17.Randall, J. R., Doku, D., Wilson, M. L., \&Peltzer, K. (2014). Suicidal Behaviour and Related Risk Factors among School-Aged Youth in the Republic of Benin. PLoS ONE, 9(2), e88233. http://doi.org/10.1371/journal.pone.0088233 http://journals.plos.org/plosone/article?id=10.1371/journal.pone.0088233

18.Ziaei, R., Viitasara, E., Soares, J., Sadeghi-Bazarghani, H., Dastgiri, S., Zeinalzadeh, A. H., ... Mohammadi, R. (2017). Suicidal ideation and its correlates among high school students in Iran: a cross-sectional study. BMC Psychiatry, 17, 147. http://doi.org/10.1186/s12888-017-1298-y https://bmcpsychiatry.biomedcentral.com/articles/10.1186/s12888017-1298 Suicidal Ideation in Relation to Self-Esteem among University Students

19.Cederbaum JA ${ }^{1}$, Gilreath TD ${ }^{2}$, Benbenishty R ${ }^{3}$, Astor RA ${ }^{2}$, Pineda D², DePedro KT ${ }^{4}$, Esqueda MC ${ }^{5}$, Atuel H². Wellbeing and suicidal ideation of secondary school students from military families. J Adolesc Health. 2014 Jun;54(6):6727. doi: 10.1016/j.jadohealth.2013.09.006. Epub 2013 Nov 17.

20. Yang H, Ran G, Zhang Q, Niu X. The Association between Parental Attachment and Youth Suicidal Ideation: A Three-Level Meta-analysis. Arch Suicide Res. 2021 Dec 29:1-26. doi: 10.1080/13811118.2021.2020192. Epub ahead of print. PMID: 34964432. 